\title{
COMMENTAIRES SUR LE PREMIER CODE DES LOIS DE HUAHINE: E TURE NO HUAHINE (1822-1823)
}

\author{
Corinne Raybaud ${ }^{*}$
}

Le 5 mars 1797 débarquèrent du «Duff», les premiers missionnaires de la London Missionary Society. Ils commencèrent l'évangélisation des populations au début du XIXème siècle, tout d'abord à Tahiti et Moorea, puis dans les îles-sous-le-vent. Avec la progression du christianisme, des pans entiers de l'ancienne religion s'effondraient et les repères de la société se dissolvaient lentement. Le roi Pomare II sut tirer le meilleur parti de l'influence des missionnaires pour accroître son pouvoir sur des populations jusque là soumises à de nombreuses autorités. Les missionnaires se servirent de l'appui des Pomare pour réaliser leur évangélisation. Rapidement la nécessité apparue de rédiger des codes de lois, sorte de codes de bonne conduite qui feraient pendant dans le domaine profane à la Bible que les missionnaires faisaient découvrir en langue vernaculaire aux populations. Ces codes relatent les derniers aspects de la coutume, dans une société de tradition orale en plein bouleversement Ainsi furent promulgués par le roi Pomare II en 1819 les 19 articles du premier code de lois en Polynésie: le code E Ture ${ }^{1}$ No Tahiti. L'année suivante, en 1820 le roi Tamatoa aux îles-sous-le-vent proclama les 25 articles du code E Ture No Raiatea e no Tahaa e no Porapora e no Maupiti... En 1823, la reine de Huahine, Teri'itaria fille de Tamatoa roi des îles-sous-le-vent et belle-sœur et concubine de Pomare II promulgua le premier code de lois de l'île de Huahine réalisé par des missionnaires et quelques chefs de l'île. Il comportait 30 articles et représentait la législation la plus aboutie en ce début de XIXème siècle en Polynésie.

En 1819 et 1820, les îles de Tahiti et de Raiatea se dotèrent des premiers codes de lois écrits qui leur permirent de construire une nouvelle société fondée sur des principes

* Docteur en Histoire contemporaine (Paris X Nanterre), Docteur en Droit (Bordeaux IV Montesquieu). Article extrait de la thèse de doctorat en droit: «De la coutume à la loi dans les archipels de Polynésie Orientale de 1767 à 1945» soutenue en octobre 2000, à l'Université Montesquieu Bordeaux IV.

1 Le mot "ture" viendrait du sanscrit et signifie "loi". 
juridiques issus en partie de la coutume et pour la plupart importés d'Europe. Ces codes rédigés en langue polynésienne par les missionnaires anglais de la LMS furent accessibles à tous.

L'île de Huahine située dans le groupe des Îles-sous-le-Vent, à quelques dizaines de milles marins dans l'est de Raiatea, était au cours des années 1820 dirigée par la reine Teri'itaria, fille du roi Tamatoa de Raiatea et concubine du roi Pomare II de Tahiti, dont elle était aussi la belle-sœur.

La reine vivait habituellement à Tahiti auprès de Pomare II, mais en 1821 le missionnaire William Ellis et quelques chefs de Huahine vinrent la trouver à Matavai où résidait Pomare II et lui demandèrent de revenir habiter à Huahine. Il fut décidé qu'après les grandes réunions annuelles qui se tenaient au mois de mai, elle pourrait les accompagner. $^{2}$

Quelques années après la mise en place des codes de lois de Tahiti et de Raiatea, l'île de Huahine se dota d'un code de lois écrit sous le nom: E Ture No Huahine.

\section{LE CODE E TURE NO HUAHINE DE 1822-1823}

Il fut tout naturel pour cette reine de se doter d'un code de lois, à l'image de ceux de ses plus proches parents de Tahiti et Raiatea. Ainsi, durant son séjour à Tahiti, W. Ellis s'installa chez un autre missionnaire $\mathrm{H}$ Nott, et ils passèrent toutes leurs journées avec quelques chefs de Huahine à préparer le code de cette île.

Ils s'efforcèrent de corriger les défauts des codes déjà publiés à Tahiti et Raiatea W Ellis écrivait: ${ }^{3}$

...j'étais très désireux que leurs lois soient rédigées avec le plus grand soin et voulait profiter de M. Nott, grand connaisseur des indigènes, et de ses observations concernant l'effet des lois sur les populations de Tahiti et Eimeo ( Moorea )...

2 Ellis W, A la recherche de la Polynésie d'autrefois, (Paris, 1972) 560.

3 Ellis W A la recherche de la Polynésie d'autrefois, supra n 2, 561. 
M Nott affirmait que les plus grands défauts qu'il avait observés provenaient du pouvoir mis entre les mains des magistrats ${ }^{4}$ de choisir les châtiments réservés aux coupables.

Ce pouvoir discrétionnaire faisait qu'un même crime était suivi de punitions différentes selon les magistrats et selon les régions. Pour remédier à cela, la punition à infliger devrait être jointe ${ }^{5}$ à la défense portée. Les lois on pouvait l'espérer, auraient ainsi une influence plus efficace.

On remarque ici la volonté des missionnaires d'améliorer ce qu'ils avaient préalablement effectué et ce dans un souci de bonne administration de la justice.

William Ellis poursuivait ses considérations en écrivant: ${ }^{6}$

Un autre sujet important concernait les ressources du gouvernement et les moyens de subsistance à fournir au roi et aux chefs. Sur ce sujet Pomare s'était refusé de légiférer, préférant demander des provisions à la population selon ses besoins plutôt que de recevoir régulièrement une partie des produits de la terre. Les droits des propriétaires étaient ainsi encore incertains et celui qui se montrait habile à cultiver, n'était pas sûr de récolter le fruit de son travail. Tout cela éclatait alors au milieu de cette affaire où le roi de Tahiti, dans son désir de payer le navire acheté en son nom, avait ordonné de nombreuses et fortes réquisitions de porcs auprès des chefs. Après avoir interdit à quiconque de vendre aux capitaines de navires ou à d'autres personnes des produits amenés pour l'échange, il avait réclamé pour lui-même des objets et n'offrait en contrepartie que des articles d'une valeur minime.

Pour remédier à cela, plusieurs lois avaient été ajoutées à celles préparées pour Huahine, et une certaine taxe, une sorte de capitation fut proposée. Un article de la loi fixait la part des

4 Les pouvoirs du juge étaient très étendus; ils soulèvent le problème de l'arbitraire. «Avant d'être un thème de polémique d'autant plus volontiers abordé que son nom seul prête à tous les amalgames, l'arbitraire est une notion juridique. Dans l'évolution générale de notre droit pénal, il domine une longue période (les trois derniers siècles de l'Ancien Régime) et s'oppose tant au principe médiéval de fixité qu'à l'expérience révolutionnaire des disciples de Beccaria. On sait combien fut brève cette dernière tentative. Par contre le système médiéval, dans lequel la peine fixe est certes un archaïsme résiduel mais aussi un moyen de lutter contre l'arbitraire seigneurial a longtemps refusé de confier au juge des pouvoirs étendus. L'arbitraire s'impose définitivement au XVI ème siècle... Jusqu'à la fin de l'Ancien Régime, la doctrine enseigne unanime que «quoique les peines soient pour la plupart arbitraires en France, les juges n'en peuvent pas inventer de nouvelles; ils doivent suivre celles qui sont reçues par les ordonnances ou par l'usage». S'il ne doit pas aller à l'encontre des usages, à plus forte raison l'arbitraire ne peut-il s'exercer que dans le respect de la loi.» Laingui A Lebigre A Histoire du droit pénal, T1, Paris, p 129-130.

5 «Réclamer la peine fixe, c'est aussi dénier au juge, qui ne devrait plus être que «la bouche qui prononce les paroles de la loi» (Montesquieu Esprit des Lois, XI, 6), le pouvoir d'appréciation sans lequel le droit classique ne pouvait se concevoir.» Laingui A Lebigre A supra n 4, 129.

6 Ibid. 
produits de l'île que chaque individu devait fournir pour l'usage du roi et pour le chef du district dans lequel il résidait. Le reste demeurait intangible et à l'usage du propriétaire qui pouvait en disposer à son gré.

Le traitement des coupables entre leur arrestation et leur jugement avait été aussi réglé. Tels furent les principaux additifs faits au code de Huahine.

L'institution d'un jury avait été introduite dans les lois de Raiatea. Les modifications furent approuvées par les chefs venus de Huahine et montrées par eux à Teri'itaria qui donna son parfait accord à l'adoption de ces lois à Huahine.

Après ce séjour à Tahiti, W Ellis et les chefs s'en revinrent à Huahine afin de mettre en vigueur ce nouveau code approuvé par la reine, les chefs et rédigé en fonction des erreurs passées, en attendant le retour de la reine qui ne voulait pas que son absence retarde la publication des lois.

Le code $E$ ture No Huahine est plus riche que les précédents. Il contient 30 lois, au lieu de 19 pour celui de Tahiti et 25 pour celui de Raiatea, ainsi qu'un additif qui peut ressembler à un code de procédure comportant 4 articles pour les juges principaux, 6 pour les juges subordonnés, 4 concernant le jury, 2 sur les messagers des magistrats et enfin 8 sur les directives données pour les juges et le jury.

Ce code comme les deux précédents est d'abord répressif puisque les articles en matière pénale représentent le plus grand nombre, soit plus de la moitié. Viennent ensuite les articles en matière civile, soit environ une dizaine. Enfin, on peut rassembler les articles restants dans une matière qui se situerait à mi-chemin entre le droit pénal et le droit administratif.

Voici les titres des différentes lois du code E Ture No Huahine de 1822-1823:

- Loi I - Concernant le meurtre.

- Loi II - Concernant le vol.

- $\quad$ Loi III - Concernant les cochons.

- $\quad$ Loi IV - Concernant les biens volés.

- Loi V - Concernant les biens perdus.

- Loi VI - Concernant l'achat, la vente et l'échange.

- Loi VII - Concernant la non observance de la journée du Sabbat.

- Loi VIII - Concernant la rébellion ou l'incitation à la guerre.

- Loi IX - Concernant la polygamie. 
- Loi X - Concernant une femme précédemment abandonnée.

- Loi XI - Concernant l'homme marié et la femme mariée.

- Loi XII - Concernant (le divorce) le bannissement de l'homme et le bannissement de la femme.

- Loi XIII- Concernant le refus de nourrir son épouse.

- Loi XIV - Concernant le mariage.

- Loi XV - Concernant la fausse accusation.

- Loi XVI - Concernant la sodomie.

- $\quad$ Loi XVII - Concernant le viol pendant le sommeil.

- Loi XVIII- Concernant l'étranglement de la femme, dans l'intention de la violer.

- Loi XIX - Concernant le concubinage.

- $\quad$ Loi XX - Concernant l'ivresse.

- Loi XXI - Concernant les dommages causés par les chiens ou les porcs.

- Loi XXII - Concernant les sangliers et les cochons errants.

- Loi XXIII - Concernant la conspiration.

- Loi XXIV - Concernant l'homme qui fait connaître la conspiration.

- Loi XXV - Concernant le fait de monter aux arbres pour y prendre de la nourriture.

- Loi XXVI - Concernant les tributs à fournir au roi et aux chefs de districts.

- Loi XXVII - Concernant le tatouage.

- $\quad$ Loi XXVIII - Concernant les voyages avec une très nombreuse compagnie.

- Loi XXIX - Concernant les juges et les magistrats.

- Loi XXX - Concernant les nouvelles lois.

- $\quad$ Lois pour les juges, le jury et les messagers ou officiers de paix.

- $\quad$ Lois concernant les juges principaux. ( 4 articles )

- $\quad$ Lois concernant les juges et les magistrats subordonnés. (6 articles)

- $\quad$ Lois concernant le jury. (4 articles)

- Lois concernant les messagers des magistrats ( ou officiers de paix ). ( 2 articles ) 
Ce code préparé principalement par William Ellis fut partiellement traduit par lui en anglais et retranscrit en français dans l'ouvrage: A la recherche de la Polynésie d'autrefois publié par la Société des Océanistes, du Musée de l'Homme à Paris.

L'original du code E Ture No Huahine en reo ma'ohi était conservé depuis le XIXème siècle au Public Record Office de Londres. Nous avons pu obtenir une copie de l'original que nous avons fait traduire ${ }^{7}$ afin de vérifier la traduction partielle de ces articles fournis par Ellis. Nous avons pu constater que les titres de certaines de ces lois n'étaient pas conformes aux faits incriminés: les lois XVI, XVII, XVIII et XIX n'avaient pas été traduites ou l'avaient été de façon très approximative.

Par exemple la loi XVI Concernant la sodomie fut traduite «Concernant le crime» sans plus de précision.

La loi XVII fut intitulée par le missionnaire «Concernant la séduction», alors qu'il $\mathrm{s}^{\prime}$ agissait de la loi en reo ma' $\mathrm{ohi}^{8}$ «No te mafera» qu'il n'était pas sensé ignorer et qui concernait le viol d'une femme pendant son sommeil.

La loi XVIII fut intitulée par Ellis «Concernant l'enlèvement», ${ }^{9}$ alors qu'il était question d'étranglement d'une femme avec intention de la violer. ${ }^{10}$ Enfin, la loi XIX intitulée par le missionnaire «Concernant l'impudicité» a été traduite par «Concernant le concubinage»

7 Cette traduction a été réalisée avec l'aide de professeurs de reo ma'ohi, et du Service des Archives territoriales pour les lois XVI et XIX.

8 Il n'existe pas en langue reo ma'ohi d'accent circonflexe mais une barre au-dessus de certaines voyelles. De plus, sur l'original aucun accent, ni apostrophe n'apparaît entre, devant ou sur les voyelles.

9 Outre, la pudeur du missionnaire à employer le terme exact de viol, on peut peut-être trouver une explication à cela. «Si les auteurs coutumiers à la différence des rédacteurs du Code pénal, n'ont aucune fausse pudeur à définir le viol, leur terminologie est toutefois décevante: pas plus que la jurisprudence ils n'emploient le mot «viol», mais n'hésitent pas à parler de «rapt» en décrivant un viol; alors que les deux incriminations semblent bien distinguées dès le XIIIème siècle: Etablissements de Saint-Louis, I, 27: «Raz, si est fame efforcier.»

Beaumanoir lui-même, qui donne une définition satisfaisante du viol, finit par une incompréhensible confusion: «Femme esforcier si est quant prend à force charnelle compagnie à femme contre la volonté de la femme et sûr qu'elle a fait ce qu'elle a pu pour se défendre.» (\$ 829) Pour le cas très différent où «aucuns fortraient (enlèvent) les femmes, les filles ou nièces d'autrui ou celles qui sont en leur garde...», il envisage (\$ 926) deux hypothèses, celle d'un départ volontaire de la femme et celle d'un départ contraint (qui n'implique pas la présomption de «compagnie charnelle») et il conclut par «L'en apele rat (rapt) femme esforcier.» Peut-être veut-il suggérer qu'en pratique l'enlèvement n'est souvent que l'acte préparatoire d'un viol ?» Laingui A Lebigre A Histoire du droit pénal, T1, Paris, p 159.

10 En reo ma'ohi « No te 'u umi vahine». 
On constate que le missionnaire a traduit à dessein, de façon évasive ou poétique, probablement parce qu'il les trouvait bien trop choquants, des actes qui faisaient partie des coutumes polynésiennes, en matière de comportement sexuel.

\section{EN MATIÈRE PÉNALE}

Les lois ayant pour objet la répression des crimes et délits sont les plus nombreuses. Parmi celles-ci, le meurtre et les agressions sexuelles ${ }^{11}$ se trouvent en tête, puis viennent les lois qui doivent sanctionner la rébellion et la conspiration; enfin l'on trouve encore des lois concernant des délits divers tels que le vol, l'ivresse, le tatouage...

\section{A Le meurtre et les agressions sexuelles}

Ces lois sur le meurtre et les agressions sexuelles reprennent comme base les codes précédents, ${ }^{12}$ avec toutefois des apports intéressants qui faisaient de ce code le plus approfondi de ceux jusque-là publiés.

\section{Le meurtre}

La loi I du code E Ture No Huahine édicte:

Si les parents tuent leurs enfants, ou des enfants non encore nés, si ce ne sont pas les parents, la famille, si ce n'est pas elle, un étranger ou toute personne qui commettra un meurtre délibérément, sera punie et sera envoyée dans une terre lointaine, inhabitée, telle que l'île de Palmerston. Ils seront ${ }^{13}$ laissés ainsi jusqu'à leur mort et ne reviendront jamais chez eux.

Comme à Tahiti et à Raiatea, l'infanticide, l'avortement ${ }^{14}$ et tout homicide volontaire étaient sanctionnés par la peine capitale. Toutefois, dans ce code de Huahine le retour à la coutume du bannissement respectait la coutume des lieux.

Dans le cas de la loi I, le code sert à rédiger la coutume orale, et n'impose pas la coutume anglaise de la pendaison.

11 Sous l'Ancien Régime, l'indifférence relative en matière d'attentats aux mœurs fait place à la répressivité pour deux crimes, le viol et le rapt, dont la violence est l'un des éléments constitutifs. Laingui A Lebigre A supra n 9, 159.

12 Soit le code E Ture No Tahiti de 1819, communément appelé Code Pomare, et le code E Ture No Raiatea, Tahaa, Pora Pora de $1820 \ldots$ appelé aussi Code Tamatoa, du nom du roi de ce groupe d'îles.

13 Les criminels.

14 L'édit de février 1556 pris par Henri II en matière d'avortement et d'infanticide, après avoir donné les éléments constitutifs du crime, déclare: «... soit telle femme tenue et réputée avoir homicidé son enfant et, par réparation punie de mort et dernier supplice, et de telle rigueur que la qualité particulière du cas le méritera.». Laingui A Lebigre A supra n 9, 130. 
L'île choisie comme lieu de bannissement était l'île de Palmerston, située à plusieurs milliers de kilomètres à l'ouest de l'archipel de la Société et des Iles Cook. La reine Teri'itaria fit preuve de volonté et de courage en ne suivant pas l'exemple de son royal amant Pomare II et de son père Tamatoa, en affirmant sa détermination à respecter la coutume ancienne.

D'autre part, face au mécontentement de la population à propos de la peine de mort par pendaison, il était plus judicieux de reconsidérer le châtiment. ${ }^{15}$

Le missionnaire William Ellis qui contribua largement à la rédaction de ce code apporta lui-même ces commentaires: 16

Dans le premier code des lois de Tahiti et de Raiatea, la peine de mort punissait le meurtre, la sédition et la trahison. Mais les lois de Huahine et des îles dépendantes n'admettaient la peine de mort pour aucun crime.

Dans la première loi qui interdisait le meurtre et toutes les formes d'infanticide, le châtiment appliqué à ces crimes au lieu de la mise à mort, était le bannissement pour la vie dans l'île Palmerston ou dans une autre île inhabitée. ${ }^{17}$ C'était la conséquence de notre intervention personnelle... L'existence d'un certain nombre d'îles inhabitées apparaît comme donnant une réponse à toutes les fins de la justice publique...

La communauté serait préservée d'un futur délit de la même manière que si le criminel avait été exécuté et nous avions la ferme conviction qu'une vie dans la solitude perpétuelle et un travail obligatoire seraient considérés par beaucoup comme intolérables et même plus affreux qu'une mort rapide...

La peine de mort par pendaison ne fut pas appliquée dans l'île de Huahine.

15 Le bannissement sur la lointaine île de Palmerston était équivalent à la peine de mort.

16 A la recherche de la Polynésie d'autrefois (Paris, 1972) 562.

17 «En l'absence de prison répressive, le bannissement a constitué jusqu'au XVIème siècle le seul moyen de débarrasser la contrée d'un individu dangereux sans cependant le tuer...Cependant, hérité du morcellement féodal, où chaque justice était en quelque sorte autarcique, le bannissement présentait un inconvénient évident: prononcé seulement «hors du ressort», il ne supprimait pas la délinquance; il ne faisait que la déplacer; sans doute même l'aggravait-il puisque le banni, privé de toute attache, ne pouvait guère s'adapter ailleurs...» Carbasse J M Introduction historique au droit pénal, ibid 223-224. Le bannissement dans une île lointaine et inhabitée telle l'île de Palmerston résolvait ce problème. 


\section{Les agressions sexuelles ${ }^{18}$}

Elles étaient probablement nombreuses et le code leur consacra trois lois.

La loi XVI «Concernant la sodomie», ${ }^{19}$ condamnait les coupables à l'exil pour la vie ou à des travaux forcés pendant sept ans. Dans le code de 1820 à Raiatea, seul le bannissement sanctionnait ce crime; deux ans plus tard à Huahine il était possible que les travaux forcés remplacent le bannissement à vie sur une île lointaine.

Deux lois furent consacrées au viol :20 la loi XVII et XVIII.

La loi XVII «Concernant le viol pendant le sommeil»:

Lorsqu'un homme viole une femme pendant son sommeil, qu'elle s'en aperçoive et que l'acte ait été commis sans son consentement, il y a crime. Le coupable subit une peine de travaux forcés d'un an (construction de route, ou de clôture), les bénéficiaires sont le roi et le chef de district.

Dans la formulation de cette loi, pour que le crime de viol existe, il fallait que la victime s'en aperçoive et que l'acte soit commis sans son consentement. S'il s'agissait d'un viol et de plus au cours du sommeil de la femme, il y a peu de chance qu'il y ait consentement; quant à ce qu'elle s'en aperçoive, l'on reste dubitatif.

Enfin, si ces deux conditions étaient réunies, fallait-il encore reconnaître le coupable et le dénoncer (ce qui n'était pas mentionné dans la loi). Une fois condamné, le coupable devait accomplir une peine d'un an de travaux forcés, ce qui était fort peu, si l'on compare cette peine à la précédente qui condamnait à l'exil ou à sept ans de travaux forcés.

Si elle ne s'en apercevait pas, il n'y avait pas crime, alors qu'il avait pourtant été commis à la faveur de sa somnolence ou encore comme il était étrangement précisé, si elle

18 «Rien n'étant plus tributaire d'une époque et d'une civilisation données que la notion de (bonnes) «mœurs», il n'est pas étonnant que l'ancien droit pénal présente, notamment sous l'influence de l'Eglise et de la morale chrétienne, d'importantes différences avec le droit actuel. L'homosexualité, même entre adultes consentants, ainsi que les rapports sexuels avec un animal (bestialité) ont toujours été passibles de mort depuis le Moyen Age jusqu'à la Révolution. Par contre, l'outrage public à la pudeur, objet du célèbre article 330 du Code pénal est totalement inconnu de l'ancien droit.» Laingui A Lebrigre A Histoire du droit pénal, T1, Paris, 158.

19 La sodomie était toujours sanctionnée par la mort sous l'Ancien Régime.

20 Dans tout le droit coutumier on retrouve les mêmes éléments constitutifs: une conjonction physique imposée de force par un homme à une femme qui a vainement tenté de s'y soustraire. Ex. T.AC Bretagne: «Si aucun force femme... et eut sa compagnie par force et oultre sa volonté.» Il s'agit donc pour l'accusation de rapporter une double preuve, celle des rapports physiques et souvent plus difficile, celle de la résistance de la femme. Laingui A Lebrigre A, supra n 18, 160. 
ne s'en apercevait pas, c'était qu'elle y avait peut-être consenti, auquel cas il n'y avait pas crime.... ${ }^{21}$

Nous nous trouvons ici en face de la délicate question du consentement de la victime. Ainsi, l'infraction apparemment commise se trouve dissoute par le consentement de la victime, en supposant qu'il soit indiscutable et donné par une personne capable.

Si le consentement porte sur une valeur disponible, l'infraction ne disparaît pas, elle n'a jamais existé. Il arrive qu'une infraction consiste dans l'atteinte à un droit dont la victime à la possibilité de disposer. Cela se produit, quoique rarement, pour des infractions contre les personnes, lorsqu'un individu consent à des jeux érotiques: il ne peut y avoir délit $\mathrm{d}^{\prime}$ atteinte sexuelle ni viol.

Encore faut-il, comme on l'a déjà noté, qu'il y ait preuve du consentement et qu'il émane d'une personne juridiquement capable. Mais si ces conditions sont réunies, il n'y a pas d'infraction.

Il n'y a pas d'infraction parce que le texte incriminateur supposait l'absence de consentement. En sa présence, les faits commis échappent aux prévisions du texte incriminateur. On ne doit donc pas parler d'un fait justificatif qui dissout une infraction par ailleurs commise. Ici, faute de texte, il n'y a pas du tout d'infraction. ${ }^{22}$

21 Sous l'Ancien Régime, la victime d'un viol devait apporter la preuve de celui-ci en se soumettant à une expertise médicale et elle devait pouvoir prouver son refus de l'acte, matérialisé par les efforts tentés contre l'agresseur. «Le problème de la preuve atteint ici son point le plus délicat et provoque en ancien droit un conflit évident entre la tendance générale à la répressivité et un antiféminisme non moins général, tant chez les auteurs coutumiers que dans la doctrine classique ou même chez les juges... Ces derniers hésitaient à prononcer la peine capitale dans des cas où les pseudo-victime auraient d'abord consenti et ensuite porté plainte dans la crainte des conséquences... Le témoignage est souvent impossible par la nature même du crime. Restent les indices, parmi lesquels le fait que la victime a crié et appelé au secours... les traces de lutte... En droit coutumier comme en droit classique, le viol est passible de mort. En fait, la peine capitale n'est prononcée - sans doute à cause des difficultés de preuve- que pour les viols de fillettes âgées de moins de 10 ou 12 ans, ou s'il s'y est ajouté un abus d'autorité. Mais quand la peine de mort est infligée, le mode d'exécution en est souvent aggravé par rapport à la pendaison ou à la décapitation de droit commun. Au Moyen Age, le condamné est souvent traîné et pendu: Boutillier, I, 39: «et par la coutume mêmement du royaume de France, ils doivent être traînés jusques à la justice et pendus tant qu'ils soient morts et étranglés.»... Toutefois certaines coutumes connaissent la grâce par mariage subséquent: si le coupable offre d'épouser sa victime, il échappe à la sanction. Encore fallait-il que le mariage fût juridiquement possible et que la victime acceptât. A partir du XVIème siècle, c'est la peine de la roue ou du feu que l'on trouve pour les viols d'enfants... En l'absence de ces véritables circonstances aggravantes que constituent l'âge de la victime ou la qualité de l'auteur, on retient plutôt la tentative de viol qui est le plus souvent sanctionnée par la peine des galères à temps ou à perpétuité.» Laingui $\mathrm{A}$ Lebrigre $\mathrm{A}$, Histoire $d u$ droit pénal, T1, Paris, 160-161.

22 Soyer JC Droit pénal et procédure pénale (Paris, 1994) 125. 
Cette pratique devait être fréquente et avoir entraîné quelques abus, pour nécessiter la rédaction d'une loi.

La loi XVIII «Concernant l'étranglement de la femme, dans l'intention de la violer» prévoit:

Lorsqu'un homme étrangle une femme dans la brousse ou un endroit isolé et qu'il commet sur elle un viol sans son consentement ${ }^{23}$, si celle-ci appelle au secours, le coupable a commis un délit grave. Il sera banni et exilé sur une autre île ou puni à des travaux forcés pendant trois ans.

L'on retrouve ici l'esprit de la loi précédente. Le rédacteur de la loi semblait se sentir obligé de préciser si le viol était avec ou sans le consentement de la victime, ce qui tendrait à imaginer que beaucoup d'actes sexuels ressemblaient à des viols, mais que certains devaient être consentis.

Il était curieux de préciser l'absence ou non de consentement, alors que la scène se déroulait dans la brousse ou un endroit isolé, et qu'en plus un étranglement était nécessaire pour immobiliser la victime.

Mais il semblerait, d'après la rédaction de la loi, que pour que l'on puisse conclure au crime il fallait encore que la victime appelle au secours. ${ }^{24}$ Ainsi, ne suffisait-il pas qu'elle

23 «Traditionnellement, la contrainte physique était assimilée à la force majeure, opinion encore en vigueur. L'absence totale de volonté criminelle propre exemptait de toute peine l'auteur de l'acte. Cette solution qui n'impute pas le crime d'adultère à la femme mariée victime d'un viol existait déjà en droit romain. Les canonistes l'avaient approuvée en déclarant qu'une femme violentée ne perd pas sa pudicita. Tous les auteurs, les coutumes ont par la suite répété cette décision, sans d'ailleurs trop s'étendre sur les circonstances qui devaient accompagner la contrainte pour rendre acceptable l'excuse invoquée par la femme.» Laingui A Lebrigre A Histoire du droit pénal, Ibid, 79.

24 L'explication se trouve peut-être dans le Deutéronome. «Suivant Deutéronome (XXII, 25-27), il fallait que la victime eût crié au secours. Curieusement, c'est au XVIIIème siècle que la question a été examinée avec le plus de détail. Voici ce qu'en écrit le jurisconsulte Fournel, dans son Ttraité de l'adultère (1778), cité par Chauveau-Hélie, au milieu du XIX ème siècle comme la doctrine la plus experte sur la matière: «La loi a prévu le cas où des hommes forcenés parviendraient, par la violence, à celui de tous les actes, qui a le plus besoin du consentement et de l'accord des deux parties. Dans ce cas, elle impose silence à la réclamation du mari et prononce l'absolution de la femme, par la raison que le cœur n'est pas complice de la souillure du corps...Mais est-il vrai qu'une femme puisse de bonne foi se trouver exposée à invoquer cette exception ? Quelle que soit la supériorité des forces d'un homme sur celles d'une femme, la nature a fourni à celle-ci des ressources sans nombre pour éluder le triomphe de son adversaire et le courage de la vertu lutte avec succès contre les attaques de la brutalité. La sagesse qui succombe est presque toujours une demi-sagesse qui travaille à sa propre défaite... Si c'est à la ville que la violence a été exercée la femme n'en a-t-elle prévenu les effets en appelant au secours ? (...)» Laingui A Lebrigre A Ibid, 79-80. 
soit agressée sexuellement dans un endroit isolé, qu'elle soit en partie réduite au silence par un étranglement, il fallait que pour caractériser le crime, elle appelle au secours.

Tout cet enchaînement de circonstances nécessaires, pour incriminer le coupable, nous amène à envisager deux hypothèses, soit la brutalité des mœurs faisait que des jeux sexuels se terminaient parfois mal et conduisaient la partenaire qui avait refusé son consentement à appeler au secours pour échapper ou se plaindre d'assauts non souhaités; soit les rédacteurs ${ }^{25}$ guidés par leurs préjugés concluaient dans beaucoup de cas à la provocation des femmes ou à leur consentement, tant qu'elles n'appelaient pas au secours.

La punition était sévère puisqu'il s'agissait d'exil ou de travaux forcés pendant trois ans.

\section{B La rébellion et la conspiration}

Comme dans les deux premiers codes, rébellion et conspiration étaient des sujets de préoccupation pour les rois et détenteurs du pouvoir dans les districts.

\section{La rébellion}

La loi VIII «Concernant la rébellion ou l'incitation à la guerre» édicte:

L'homme qui voudra provoquer une guerre, qui fera circuler secrètement de fausses nouvelles, qui excitera secrètement les sentiments de la population contre le roi ${ }^{26}$ légal ou qui emploiera tout autre moyen pour provoquer une rébellion, cet homme passera devant un tribunal et s'il est accusé d'avoir provoqué une rébellion, il sera envoyé dans son propre district ou dans son île, mais si de nouveau il provoque une rébellion, il aura à construire un furlong (201 m) de route. S'il recommence à nouveau, il sera exilé dans une île lointaine, l'île de Palmerston par exemple et il ne reviendra que si le roi le désire.

25 La rédaction des codes fut effectuée par des hommes: des missionnaires et des chefs. Dans la note précédente, les jurisconsultes du XVIIIème siècle n'étaient pas moins anti-féministes que les missionnaires et les chefs du début du XIXème siècle, en Polynésie.

26 Le crime de lèse-majesté au premier chef est le meurtre ou la tentative de meurtre commis contre le roi et sa famille, ainsi que la "conspiration contre l'Etat», c'est-à-dire contre le régime monarchique lui-même, voire contre la dynastie régnante. Un édit de François $1^{\text {er }}$ déclare coupables de lèse-majesté au premier chef «ceux qui auront ... conspiré, machiné ou entrepris contre notre personne, nos enfants et postérité ou la république (au sens latin de res publica: bien commun) de notre royaume». Ferrière dit que la lèse-majesté au premier chef est «la conspiration ou conjuration contre l'Etat ou la personne du Prince, pour le faire mourir soit par force et violence d'armes, poison ou autrement.» Le répertoire de Guyot et Merlin ajoute à la personne du prince celle de ses enfants. Il s'agit donc d'abord du régicide au sens large du terme. On peut y joindre les fauteurs de trouble qui tendent à provoquer le remplacement de la dynastie régnante par une famille rivale... Par conspiration ou conjuration on désigne même l'acte d'un individu isolé... Laingui A Lebrigre A, Histoire du droit pénal, Ibid, 200-201. 
On remarque que la peine était graduée. ${ }^{27}$ Ainsi à la première condamnation le coupable était renvoyé dans son lieu d'origine. Sa peine prenait donc la forme d'une extradition.

S'il récidivait, il était condamné à une peine de travaux forcés d'intérêt général.

$S^{\prime}$ il persistait dans la conspiration et la rébellion, ce multi-récidiviste ne bénéficiait plus de sursis. Il voyait sa peine devenir très lourde puisque c'était le bannissement dans l'île de Palmerston qui lui était infligé.

Le code de Huahine était beaucoup plus empreint de sérénité et de modération ${ }^{28}$ que les deux précédents qui condamnaient au bannissement, pour le même crime. ${ }^{29}$

\section{La conspiration}

D'autres lois traitaient de la conspiration et des fauteurs de trouble; il s'agissait des lois XXIII, XXIV et XXVIII.

Les lois XXIII et XXIV «Concernant la conspiration» ${ }^{30}$ et «Concernant l'homme qui fait connaître la conspiration» ont déjà été traitées dans le code E Ture No Raiatea... de 1820.

27 Il n'existe pas de véritable échelle des peines en ancien droit, ni au Moyen Age où elle aurait pu au moins, faute d'une échelle générale inconcevable dans une organisation judiciaire aussi peu centralisée, se trouver au sein de chaque ensemble coutumier, ni en droit classique malgré la tentative esquissée par l'Ordonnance de 1670. A l'article 13 de son titre XXV, celle-ci porte: «Après la peine de mort naturelle (par opposition à la mort civile), la plus rigoureuse est celle de la question avec la réserve des preuves en leur entier, des galères perpétuelles, du bannissement perpétuel, de la question sans réserve des preuves, des galères à temps, du fouet, de l'amende honorable et du bannissement à temps.» Ibid 125.

28 Les deux aspects les plus marquants de l'ancien droit pénal sont d'une part l'absence de sanctions moyennes, ce qui valorise à l'excès les peines les plus rigoureuses, et d'autre part un très large pouvoir d'appréciation laissé aux juges sous le nom d'arbitraire. Laingui A Lebrigre A Ibid, 125. En revanche, à Huahine dès 1822, un code plein de sérénité et de modération prenait forme.

29 Loi VIII, des codes E Ture No Tahiti et E Ture No Raiatea.

«Dans le cas de crime de lèse-majesté, en France sous l'Ancien Régime, les docteurs français considéraient que la seule pensée de ce crime en rendait coupable, quoiqu'elle n'eût été suivie d'aucun effet, à condition toutefois qu'il s'agit de lèse-majesté au premier chef. Les docteurs allèguent plusieurs arrêts portant des peines capitales contre des sujets qui avaient eu la pensée de commettre un régicide. Il s'agit d'arrêts rendus en des temps troublés, au XVIème siècle. Voici les termes de l'un d'eux, tels que les rapporte Denisart: «Le vicaire de Saint-Nicolas des Champs, pour avoir dit, en tenant un couteau: «il se trouvera encore quelqu'homme de bien comme frère Clément pour tuer le roi, et ne fût-ce que moi, a été condamné par arrêt du mercredi 11 janvier 1595, confirmatif de la sentence du Prévôt de Paris, à être pendu et étranglé; exécuté le même jour à la porte de Paris.» Laingui A Lebigre A Histoire du droit pénal T1, Paris, 51. En Polynésie, l'intention criminelle ne suffisait pas pour être condamné pour crime de lèse-majesté; en revanche l'omission de dénoncer le crime était coupable. 
En revanche la loi XXVIII intitulée «Concernant les voyages avec une très nombreuse compagnie» était attribuée aux fauteurs de trouble:

Quand un membre de la famille royale, un chef de district, ou un homme de haut rang et de grande influence décide de partir en voyage dans une autre île, par exemple de Raiatea ou Tahiti à Huahine, il faut qu'il choisisse des hommes sûrs, tels que des membres de l'Eglise ou des personnes ayant été baptisées.

Les fauteurs de trouble et les criminels devront obligatoirement rester dans leurs îles. Mais si ces voyageurs continuent à amener des hommes provoquants des troubles en débarquant à terre, les magistrats leur demanderont de ne pas troubler la paix et de ne pas se promener durant la nuit.

S'ils ne tiennent pas compte de la demande, les perturbateurs seront attachés par des cordes jusqu'au départ des personnalités; et alors seulement ils seront libérés.

Les fauteurs de trouble se manifestaient lors des déplacements des rois, de la reine ou des chefs et personnalités importantes. ${ }^{31}$ Les déplacements de nuit étaient dangereux et déconseillés, et les fauteurs de troubles qui étaient connus étaient alors attachés jusqu'au départ des voyageurs de haut rang. Il s'agissait visiblement de mesures préventives, afin d'éloigner les détenteurs du pouvoir de la secte des arioi.

\section{AUTRES DÉLITS}

Parmi ceux-ci, on trouve le vol, l'ivresse et les fausses accusations.

\section{Le vol}

Les lois II, IV, V, XXII et XXV caractérisent ce délit.

La loi II «Concernant le vol» s'inspire de la loi II des codes E Ture No Tahiti de 1819 et E ture No Raiatea... de 1820. Toutefois, celle de Huahine apporte davantage de précision, quant à la tarification de la peine, afin de limiter le pouvoir discrétionnaire du magistrat, grave défaut des deux premiers codes, auquel le missionnaire William Ellis comptait remédier.

\section{La loi II édicte:}

Si un homme vole un cochon, il devra en rendre quatre: deux pour le propriétaire, deux pour le roi. S'il n'a pas de cochons, deux pirogues simples, une pour le propriétaire, une pour le roi. S'il n'a pas de pirogue, deux balles de tissu indigène si les crocs lui sortaient de la bouche, ce qui signifiait que le cochon était adulte et de grande taille.

31 Les missionnaires qui rédigeaient ces codes n'oubliaient pas de rappeler que les rois devaient s'entourer de personnes sûres, comme eux ou des hommes ayant été baptisés. 
Chaque balle devra contenir cent toises (200 yards) de tissu, large de quatre yards. Si le cochon n'était pas adulte, cinquante toises. Si c'était un petit cochon, vingt toises. Pour le propriétaire du cochon la moitié, et l'autre moitié pour le roi.

S'il n'a pas de tissu, on donnera de l'arrow-root. Si le cochon volé était gros, quarante mesures (une mesure contenait quatre à cinq livres). Pour un cochon non adulte, vingt mesures et pour un petit, dix mesures. Pour le propriétaire du cochon la moitié et l'autre moitié pour le roi. L'arrow-root pour le roi devra être de la même grosseur que pour le propriétaire.

S'il n'a pas d'arrow-root, tout autre bien lui appartenant.

Toute chose volée devra être multipliée par quatre (le paiement): deux parts pour le roi, deux parts pour le propriétaire.

Si le voleur n'a pas de biens, il devra travailler sur les terres de la personne qu'il a volée. ${ }^{32} S^{\prime}$ il refuse, sa terre deviendra la propriété du roi et il sera obligé de devenir un vagabond pour une période illimitée. Si le roi le réhabilite, il reviendra sur ses terres, s'il ne l'est pas (réhabilité), il ne reviendra pas.

Les magistrats devront appliquer la punition pour ce crime qui se trouve dans les lois, et cette punition seulement. Les juges ne devront pas demander de paiement aux parents du voleur.

L'expérience des missionnaires acquise lors de la rédaction des deux codes précédents, et surtout l'étude de l'effet de ces codes sur les populations dans la pratique d'une bonne administration de la justice, poussèrent ces hommes de religion devenus juristes par la force des choses à apporter encore quelques améliorations à ces codes.

Une plus grande minutie dans l'échelle des peines apparaît, car dans les deux premiers codes on ne se souciait pas de la taille du cochon volé. Pour tout vol de cochon, le voleur devait rendre quatre cochons ou deux pirogues ou deux rouleaux d'étoffe, ou encore devait travailler pour la victime du vol et à défaut était réduit à l'errance après avoir perdu sa terre au profit du roi.

Le nouveau code de Huahine établissait une classification des peines pour le vol de cochon. Il s'agissait de peines pécuniaires, en l'espèce de peines privatives d'un droit d'ordre patrimonial, le droit de propriété. Cette peine était déterminée en fonction de la taille de l'animal. Ainsi, le cochon adulte volé étant un bien de plus grande valeur qu'un petit cochon, la peine était plus lourde.

32 Sous la forme d'un esclavage. 
Enfin, faute de paiement de l'amende, le coupable pouvait encourir la confiscation de sa terre ${ }^{33}$ et se trouver ainsi démuni de tout et livré à l'errance, jusqu'à une hypothétique réhabilitation due à l'usage du droit de grâce du roi. Dans ce cas, il semble que la peine était éteinte et faisait cesser la déchéance qui accompagnait la condamnation.

Toujours sur le vol de cochon, la loi XXII «Concernant les sangliers et les cochons errants» punissait tous ceux qui chassaient les cochons, qu'ils soient domestiques ou retournés à l'état sauvage, car les rédacteurs de la loi se fondaient sur le principe que nous avons déjà évoqué dans le code $\mathrm{E}$ Ture No Raiatea...: pas de cochons sans propriétaire. Ainsi, tout contrevenant se voyait infliger la peine prévue à la loi II.

La loi XXV «Concernant le fait de monter aux arbres pour y prendre de la nourriture» s'insérait dans le groupe de textes qui caractérisaient le vol.

Cette loi prévoyait:

Le fait de grimper aux arbres qui ne leur appartiennent pas, est un délit pour ceux qui le font.

De le faire en le demandant auparavant au propriétaire est autorisé.

L'homme qui vole de la nourriture dans un jardin ou dans une maison, prend ce qui ne lui est pas donné par le propriétaire de la terre. Si le propriétaire désire que le voleur soit jugé, il sera jugé et puni par des travaux. Pour de la nourriture volée dans un jardin, il devra effectuer des travaux pour le propriétaire tels que construire une barrière, la longueur devant dépendre de la valeur de la nourriture volée.

Mais si la nourriture pousse sauvagement ou n'est pas enfermée par une barrière, il devra faire quarante toises de route ou casser des cailloux sur une longueur de quatre toises.

Il semblerait donc, comme pour la loi XXII sur les cochons errants, que l'on n'ait pas le droit de capturer les animaux divaguants ou de cueillir la nourriture sauvage. Cet acte étant considéré comme un vol, les coupables étaient condamnés dans le cas de la nourriture à un travail d'intérêt général. ${ }^{34}$

Les lois IV et $\mathrm{V}$ «Concernant les biens volés» et «Concernant les biens perdus» étaient identiques aux lois IV et $\mathrm{V}$ des deux premiers codes et caractérisaient les délits de vol et de recel.

33 En Polynésie, cette peine était assez rare, à cause du lien identitaire très fort qui reliait tout individu à sa terre. En revanche, sous l'Ancien Régime la confiscation générale des biens comme peine accessoire était répandue.

34 Cette peine indique un changement considérable effectué en quelques années sous l'influence des missionnaires. Les Polynésiens avaient de tous temps vécu de cueillette et cette notion désormais associée au vol devait bouleverser les mentalités. 


\section{L'ivresse et le tatouage}

Les lois XX et XVII définissaient ces deux délits:

Concernant l'ivresse

Si un homme boit de l'alcool jusqu'à ce qu'il soit intoxiqué ${ }^{35}$ et ressent alors des troubles, les magistrats le feront attacher et quand les effets de l'alcool se seront dissipés, ils lui diront de ne plus recommencer.

Mais s'il persiste à boire de l'alcool et si les effets deviennent préjudiciables, il devra être conduit devant les magistrats et condamné à travailler à la construction d'une route de cinq toises de long et deux de large. Sinon à l'installation d'une clôture autour d'une plantation, longue de cinq toises.

Si une femme est coupable de cette faute, elle devra tresser deux grands manteaux, un pour le roi et l'autre pour le chef de district ou quarante toises de tissu, vingt pour le roi et vingt pour le gouverneur ou chef de district.

La peine était graduée en fonction de sa fréquence. La première fois, celui qui avait abusé de l'alcool était privé de liberté quelques heures, puis averti et on lui enjoignait de ne pas recommencer. Il bénéficiait alors d'une mesure de sursis. Puis lors de la récidive il était condamné à une peine d'intérêt général, de construction de route ou de clôture pour les hommes et de tressage pour les femmes.

Le travail d'intérêt général était une tradition en Grande-Bretagne et aux Etats-Unis, mais une création récente en France. ${ }^{36}$

La loi XXVII «Concernant le tatouage» édicte:

Personne ne devra se tatouer. Cette pratique doit être entièrement abolie. Elle appartient aux anciennes et mauvaises habitudes. ${ }^{37}$ L'homme ou la femme qui se feront graver des tatouages, si la chose est manifeste, seront jugés et punis. La punition de l'homme sera la suivante: il devra travailler sur une portion de route longue de dix toises pour le premier tatouage, vingt

35 La traduction littérale serait «empoisonné».

36 Cette peine a été instaurée par la loi du 10 juin 1983. Cette peine restrictive de liberté se trouve à présent prévue par l'article 131-8 du Nouveau Code Pénal. Toutefois actuellement, le prévenu peut user de son droit de refus d'accomplir cette peine et celle-ci ne peut être prononcée en son absence à l'audience, alors qu'en Polynésie c'était une peine obligatoire.

37 Pour les missionnaires qui avaient rédigé les codes, le tatouage représentait l'expression des coutumes païennes et devaient à ce titre disparaître. Dieu ayant fait l'homme à son image, il était impie que celui-ci en modifie l'apparence de sa propre volonté. 
toises pour le second, ou casser des pierres sur une longueur de quatre toises et une largeur de deux toises et il devra accomplir un autre travail pour le roi.

La punition de la femme sera la suivante: elle devra faire deux grands manteaux, un pour le roi, l'autre pour le gouverneur ou chef de district ou quatre petits, deux pour le roi, deux pour le chef de district. Sinon une longueur de dix toises de tissu indigène pour le roi et dix pour le gouverneur.

Pour l'homme ou la femme qui persisteront à se tatouer plus de quatre ou cinq fois, motifs ou ornements devront être détruits en les noircissant et les individus seront punis comme il est indiqué ci-dessus.

Le tatouage était donc formellement interdit et, pour tout nouveau tatouage, on encourait une peine de travail d'intérêt général qui augmentait avec le nombre de nouveaux tatouages, allant après cinq récidives jusqu'à la destruction des motifs. Elle se pratiquait apparemment en les noircissant, faisant donc effectuer un nouveau tatouage destiné à cacher tous les autres par l'injection d'une sorte de suie.

Cette interdiction absolue concernant le tatouage émanait des missionnaires qui voulaient ainsi faire cesser une pratique très ancienne, qui faisait partie intégrante du patrimoine culturel des anciens Polynésiens.

En quoi les tatouages les gênaient-ils dans leur œuvre d'évangélisation?

Dans les populations traditionnelles, le tatouage avait une signification magicoreligieuse et il était lié à un rite de passage ou d'intégration dans une société. Le tatouage conférait aussi une certaine puissance contre les forces maléfiques; c'était un symbole magique protecteur contre les mauvais esprits.

Pour ces croyances attachées au passé, les missionnaires voulaient faire cesser la pratique du tatouage. Leur foi en un Dieu unique devait désormais suffire à les protéger.

Mais les croyances du passé et les pratiques culturelles furent tenaces parce qu'elles étaient solidement ancrées dans les comportements.

Le tatouage était une technique d'ornementation corporelle qui revêtait des significations multiples, à la fois religieuses, sociales et guerrières. Le mot tatouage vient du mot tahitien tatau et a été pour la première fois mentionné par le capitaine James Cook lors de son premier séjour à Tahiti en 1769.

Toutefois, Samuel Wallis lorsqu'il découvrit Tahiti en 1767, décrivit la pratique du tatouage en ces termes dans sa Relation d'un voyage autour du monde: ${ }^{38}$

38 Scemla JJ, Le voyage en Polynésie, (Paris, 1994) 877. 
«Je remarquai que c'est ici un usage universel parmi les hommes et les femmes de se peindre les fesses et le derrière des cuisses, avec des lignes noires très serrées, et qui représentent différentes figures; ils se piquent la peau avec la dent d'un instrument assez ressemblant à un peigne, et ils mettent dans les trous une espèce de pâte composée d'huile et de suie qui laisse une tache ineffaçable. Les petits garçons et les petites filles au-dessous de douze ans, ne portent point ces marques; nous vîmes quelques hommes dont les jambes étaient peintes en échiquier de la même manière, et il nous parut qu'ils avaient un rang distingué et une autorité sur les autres insulaires.»

D'après le témoignage du premier Européen à Tahiti, il semblerait que le tatouage était pratiqué en signe d'initiation comme une marque de l'accession à un certain degré de maturité sociale, lors des rites initiatiques de la puberté. Il s'agissait aussi de marque d'identification de la personne et d'une appartenance à un groupe social.

D'autres navigateurs de passage en Polynésie parlèrent des tatouages. Adam Johan von Krusenstern en 1804 admira la perfection des tatouages effectués aux Iles Marquises. Il écrivait dans son Voyage autour du monde: ${ }^{39}$

Parvenus à l'âge viril, les Noukahiviens ${ }^{40}$ se tatouent tout le corps avec une perfection qui, nulle part, n'est portée à un si haut degré....Le roi, le père du roi et le grand prêtre sont les seuls que nous ayons vus tatoués de la tête aux pieds. Toutes les parties de leur corps, le visage, les yeux et même quelques endroits de la tête dont les cheveux avaient été rasés, étaient ornés de cette manière... Les femmes ne sont tatouées qu'aux mains, aux bras, au lobe de l'oreille et aux lèvres. Les gens de la classe inférieure sont beaucoup moins tatoués et plusieurs même ne le sont pas du tout. Il paraît donc que cet ornement n'appartient qu'aux personnes distinguées.

Frederick Beechey en 1826 dans sa Relation de voyage écrivit: ${ }^{41}$

Cette mode générale dans les mers du sud, outre qu'elle a d'utiles effets, peut encore être fort élégante, lorsqu'on la pratique avec goût. Les naturels des îles Gambier savent tirer de cet ornement meilleur parti qu'en tout autre pays que je sache, sans même excepter les îles Marquises.

Dans toutes les îles de Polynésie, le tatouage tenait une place importante dans la vie des insulaires. L'interdiction énoncée dans le Code de Huahine de 1822-1823 fut suivie dans les autres îles et les repères sociaux, culturels et religieux furent considérablement

39 Ibid, 877-878.

40 Habitants de l'île de Nuku-Hiva aux îles Marquises.

41 Ibid, 879 
bouleversés en quelques années. La réaction surgit avec la secte des Mamaia, qui s'insurgeait contre tous ces interdits imposés par les missionnaires.

\section{Fausse accusation et corruption}

La loi XV «Concernant la fausse accusation» précise:

L'homme qui accusera faussement un autre homme devant un magistrat, avec l'intention $\mathrm{d}^{\prime}$ amener la personne accusée devant un tribunal ${ }^{42}$ ou l'homme qui viendra faire un faux témoignage et dont le but et l'intention sont de donner un faux témoignage, sera puni; si son accusation est prouvée être fausse, la punition qu'aurait eue à subir l'accusé (s'il avait été reconnu coupable) devra être transférée au faux témoin.

Les entraves à l'exercice de la justice étaient désormais ainsi sanctionnées.

La loi XXIX «Concernant les juges» avait pour objet la corruption des magistrats. ${ }^{43}$

\section{EN MATIÈRE CIVILE}

Dans le code E Ture No Huahine de 1822-1823, réalisé sur la base des deux précédents, on trouve davantage de précisions quant aux peines qui sanctionnent l'action en responsabilité civile liée aux dégâts causés par les animaux domestiques dans une société rurale. Les lois consacrées au mariage deviennent aussi plus nombreuses.

\section{Délits et quasi-délits}

Les lois III et XXI énonçaient différents cas d'espèces ainsi que les sanctions appropriées concernant les cochons et les chiens.

A Responsabilité du fait des choses. ${ }^{44}$

Voici le contenu de la loi III «Concernant les cochons»:

42 Sous l'Ancien Régime, la dénonciation calomnieuse pouvait donner lieu dans certains cas, à des dommages-intérêts. Laingui A Lebrigre A, Histoire du droit pénal, supra n 30, 160.

43 Cette loi figurait déjà dans le code E Ture No Raiatea.

44 Sous l'Ancien Régime, les cas d'accidents causés par des animaux non surveillés étaient considérés par la justice avec une certaine clémence envers les propriétaires Ceux-ci, selon les circonstances étaient généralement tenus de la réparation des dommages, mais la poursuite était presque toujours civile...La mansuétude des décisions demeure parfois surprenante. Jousse rapporte d'après Mornac: «l'espèce d'un fermier, qui un jour de dimanche à l'heure des vêpres avait mis ses chiens hors de sa maison et à sa porte pour la garder, pendant que lui et ses valets seraient à l'office; un jeune cordelier qui vint à passer par là, fut déchiré par ces chiens: mais comme on reconnut l'innocence du fermier, il fut seulement condamné en quarante livres d'aumône envers les cordeliers de Beauvais.» Jousse, Traité de la justice criminelle de France T1, (Paris, 1771) 527. 
Si un cochon rentre dans un jardin et détruit les récoltes, aucune amende ne sera demandée à cause de la mauvaise qualité de la clôture.

Si des pierres sont jetées à un cochon et si elles le blessent ou le tuent, l'homme ayant commis cet acte devra prendre le cochon et en fournir un autre de taille égale qu'il amènera au propriétaire du cochon blessé ou tué.

S'il n'a pas de cochon il devra donner un autre bien quelconque en guise d'amende. Pour un gros cochon, vingt mesures d'arrow-root et pour un cochon plus petit dix mesures.

S'il n'a pas d'arrow-root, autant de bambous pleins d'huile de noix de coco, que de mesures d'arrow-root.

S'il n'en a pas, il devra faire un travail personnel: pour un grand cochon il devra faire vingt toises de clôture, pour un cochon plus petit, cinq toises pour le propriétaire du cochon.

Si la clôture était bonne et a été cassée (par la faim et l'obstination du cochon) et les récoltes détruites, le cochon ne sera pas tué mais saisi et attaché, les magistrats devront fixer le montant du dédommagement à remettre au propriétaire du jardin. Le propriétaire devra également réparé la clôture.

D'emblée la loi III énonce le principe du partage de la responsabilité en raison du défaut d'entretien de la clôture. Les récoltes détruites ne seront pas indemnisées par le propriétaire du cochon, parce que la garde des récoltes par une clôture en état n'a pas été correctement effectuée, la clôture étant défectueuse.

Une minutieuse tarification avait été élaborée, dans le cas où un cochon serait blessé ou tué à coups de pierres.

Enfin, si la clôture était en bon état et a été détériorée par le cochon ainsi que les récoltes, la responsabilité pèsera alors uniquement sur le propriétaire du cochon qui en avait la garde et les juges établiront le montant du dédommagement.

William Ellis apporta des commentaires à cette loi: ${ }^{45}$

Leurs cochons et leurs jardins sont parmi les sources principales de leur entretien et de leur richesse. Les animaux ne sont pas gardés dans des étables ou dans des enclos, mais pâturent en liberté dans le district; une grande partie de leur nourriture provient des noix de coco, des fruits de l'arbre à pain, des châtaignes et d'autres fruits tombés des arbres.

Durant la saison des fruits, ceux-ci étaient abondants et les cochons, bien nourris dormaient à

l'ombre; mais durant les autres saisons de l'année, ils semaient beaucoup de troubles.

45 Ellis W, A la recherche de la Polynésie d'autrefois, (Paris, 1972) 584. 
Leurs matériaux pour construire des barrières, n'étaient pas bons, et un puissant verrat affamé forçait très facilement son chemin dans le jardin et avec ses défenses et ses dents causait souvent, en un rien de temps un terrible ravage...

\section{Responsabilité du fait des animaux}

La loi XXI intitulée «Dommages causés par les chiens ou les porcs» que nous avons déjà évoquée dans le code $E$ Ture No Raiatea...décidait que des compensations équitables devaient être fournies chaque fois qu'un chien ou un cochon tuait d'autres animaux domestiques.

Mais si le propriétaire persistait à garder un animal dangereux, lors d'un nouveau dommage il était condamné au quadruple de ce qui avait été détruit (deux parts pour le roi et deux pour le propriétaire); l'animal fautif était abattu, et le propriétaire récalcitrant était sanctionné par la peine de la loi XX.

\section{Le mariage}

Plusieurs lois traitaient du mariage, de la répudiation et du concubinage. Il s'agissait des lois IX à XIV et de la loi XIX.

\section{Polygamie et abandon}

La loi IX, comme dans les deux premiers codes, luttait contre les pratiques de la polygamie. ${ }^{46}$ Le texte a été repris, mais le code de Huahine ajoutait une sanction à l'interdit; ainsi était-il prescrit: ${ }^{47}$

Quand un homme persiste à prendre une seconde femme, les magistrats considéreront que la seconde femme est séparée de lui et donneront à chacun, comme punition, des travaux à faire.

L'homme devra construire une portion de route, longue de quarante toises et large de deux et la femme devra faire deux nattes pour dormir ou bien quatre manteaux...

Les coupables étaient donc condamnés à un travail d'intérêt général.

La loi $X$ «Concernant une femme précédemment abandonnée au temps du paganisme» a été reprise en partie des codes antérieurs avec un apport concernant la peine:

46 «La tradition romano-chrétienne est monogamique. Mais Tacite raconte en effet que les grands prenaient volontiers plusieurs épouses «en raison de leur noblesse», et cet usage semble avoir perduré. Les chroniqueurs de l'époque franque rapportent en effet que les monarques eux-mêmes ne dédaignaient pas cette tradition aristocratique contre laquelle les conciles se sont élevés.» Bart J, Histoire du droit privé, (Paris, 1998) 55.

47 Deux pour le roi et deux pour le chef de district. 
L'homme et la femme qui persisteront à revenir l'un vers l'autre seront punis; la punition sera

la même que pour la neuvième loi.

Les lois IX et $X$ dans les deux premiers codes se contentaient d'énoncer des interdits sans mentionner de sanction. Celles-ci étaient livrées à l'appréciation du juge. Aussi dans le Code de Huahine, des sanctions précises furent prévues pour faire apparaître aux yeux des coupables une réalité au délit, en énonçant clairement la sanction, mais aussi comme nous l'avons déjà mentionné, afin de limiter le pouvoir discrétionnaire des juges.

\section{Adultère et répudiation}

La loi XI «Concernant l'homme marié et la femme mariée» est une reprise des deux codes précédents. Cette loi concerne le délit d'adultère et réclame une amende pour la partie offensée, en interdisant à l'offenseur de se marier durant la vie du conjoint.

La loi XII «Concernant (la répudiation) ${ }^{48}$ le bannissement de l'homme et le bannissement de la femme» était intitulée dans les premiers codes «Concernant l'abandon du conjoint».

Le code E Ture No Huahine apporte de nouveaux éclairages à ce délit. Il ne fait plus l'objet de l'exil, mais de travaux:

L'homme ne doit pas répudier sa femme parce qu'elle lui a été infidèle. Les magistrats demanderont à un tel homme de reprendre sa femme. S'il n'accepte pas la demande, il sera puni de travaux jusqu'à ce qu'il reprenne sa femme. S'il ne veut pas, ils devront rester tous les deux ainsi jusqu'à la mort de l'un deux et l'homme n'épousera pas une autre femme.

La femme qui bannira son mari sans raison aura la même loi appliquée que pour l'homme.

Mais si l'homme répudie sa femme à cause de son mauvais caractère et de sa mauvaise conduite, les magistrats demanderont à l'homme et à la femme de vivre ensemble; mais s'ils refusent, ils laisseront l'affaire; la femme ne devra pas prendre un autre mari et le mari ne devra pas prendre une autre femme.

Il seront également condamnés à des travaux jusqu'à ce qu'ils reprennent la vie commune. L'homme devra accomplir du travail sur les routes ou dans les plantations. La femme devra

48 «Déjà avant les invasions, la législation des Empereurs chrétiens n'avait pas pu s'opposer vraiment aux usages antiques favorables au divorce et les traditions germaniques y étaient d'autant moins opposées qu'elles admettaient largement la répudiation... Les lois «barbares» reconnaissent aisément que les époux puissent volontairement mettre fin à leur union (...) Chez les Wisigoths comme chez les Burgondes, les deux rédactions s'inspirant des constitutions du BasEmpire énumèrent les nombreuses causes légitimant la répudiation de la femme, voire du mari, permettent ensuite un nouveau mariage, et admettent même le divorce par consentement mutuel.» Bart J, Histoire du droit privé, (Paris, 1998) 59-60. 
accomplir des travaux de tissage et de couture. Une part du travail ira au roi et l'autre au chef de district.

Pour la première fois, la notion d'infidélité apparaît. Au fur et à mesure, on peut observer l'œuvre des missionnaires ${ }^{49}$ qui façonne les mentalités polynésiennes au fil des codes et des années en introduisant le principe de la monogamie, du mariage, de la fidélité et par voie de conséquence de l'infidélité. Ces concepts étaient envisagés auparavant à la manière polynésienne, c'est-à-dire sans exclusivité et notion de propriété, d'appartenance, d'attachement irrévocable...

Désormais, les Polynésiens qui adhéraient à la croyance d'un dieu unique devaient prendre une seule femme et ne pas réagir à une possible infidélité par la répudiation. Le divorce n'était pas possible dans cette nouvelle religion apportée par des missionnaires protestants. C'était la vertu chrétienne du pardon qui devait permettre au conjoint malheureux de ne pas répudier son épouse. L'indissolubilité ${ }^{50}$ des liens du mariage était donc affirmée.

L'homme et la femme étaient sanctionnés des mêmes peines, ce qui indiquait l'importance du rôle social joué par la femme qui pouvait aussi répudier son mari.

Il est curieux de remarquer que la répudiation pouvait avoir été justifiée au nom du mauvais caractère ${ }^{51}$ de la femme.

Les coupables étaient condamnés à un travail d'intérêt général.

Nous pouvons citer ici le commentaire de W Ellis: ${ }^{.2}$

49 «En Occident, l'Eglise a éprouvé beaucoup de difficultés à faire prévaloir l'idée qu'un mariage ne pouvait être rompu que par la mort de l'un des époux, non seulement en raison de la force des traditions et des mœurs contraires, mais aussi à cause de l'ambiguïté des textes sacrés sur lesquels reposaient sa doctrine, si bien que les conciles eux-mêmes ont d'abord adopté des positions contradictoires. Si certains textes apostoliques rejettent entièrement le divorce, deux passages de l'évangile selon Mathieu (V, 31-32 et XIX, 7-9), tout en rapportant la condamnation par le Christ de la répudiation de la femme suivie d'une nouvelle union, admettent une exception au détriment de celle qui se livre à la fornication. Aussi les Pères de l'Eglise furent-ils tout d'abord divisés au sujet de l'interprétation de ces textes qui laissaient entendre que l'inconduite de l'épouse justifiait la rupture complète du lien matrimonial à la discrétion du mari qui pourrait ensuite légitimement se remarier. Cependant, à partir du Vème siècle, Augustin qui a consacré au problème un traité particulier (De adulteriis conjugiis)fait prévaloir dans la doctrine le principe de l'indissolubilité du mariage, sans grand succès.» Bart J, Ibid, 60-61.

50 «Il faut attendre l'époque carolingienne pour que le principe d'indissolubilité, reposant sur l'idée que l'union de l'homme et de la femme reflète celle du Christ et de l'Eglise, progresse quelque peu.» Ibid, 61.

51 Peut-être cela pouvait-il aussi signifier mauvais comportement, ce qui rapprocherait de la notion de répudiation pour fornication des premiers siècles de notre ère en Occident. 
Le douzième commandement qui concerne la dissolution du mariage est un article un peu spécial... bien que l'on ne supposât pas que les travaux forcés pussent faire renaître l'affection dans les cœurs de ceux qui, malgré leur solennelle promesse de vivre ensemble pour toute la vie étaient maintenant devenus des étrangers l'un pour l'autre, cependant on pouvait penser qu'une admonestation par le magistrat et l'exposé des conséquences d'une désaffection durable pourraient engager les époux à prendre un peu davantage sur eux et à faire un effort pour vivre ensemble en paix si ce n'est avec tendresse et amour.

\section{Les obligations du mariage}

La loi XIV «Concernant le mariage» était une reprise de la même loi dans les premiers codes. Toutefois, dans la dernière partie, on pouvait noter la présence de quelques éléments nouveaux:

Quand le jour de la célébration du mariage arrivera, des personnes devront également venir comme témoins. Le missionnaire demandera alors à l'homme de prendre la main droite de la femme et lui disant:

Prends-tu cette femme pour épouse légitime et lui seras-tu fidèle jusqu'à la mort? L'homme devra alors répondre «oui» et il demandera à la femme:

Prends-tu cet homme pour époux légitime et lui seras-tu fidèle jusqu'à la mort? La femme devra alors répondre «oui».

Ensuite le missionnaire déclarera à toute la population: ${ }^{53}$

Ces deux personnes sont devenues légalement mari et femme, en présence de dieu et des hommes. L'enregistrement du mariage devra être fait par le missionnaire dans le livre des mariages et signé de lui-même, des époux et des témoins. Le mariage sera ainsi officiel.

Personne ne peut devenir secrètement mari et femme. C'est interdit.

Parmi les apports de ce code, on note la présence de témoins qui n'étaient pas mentionnés auparavant. Le missionnaire dans sa phrase rituelle de célébration du mariage exigeait des époux qu'ils prêtent serment de fidélité en se choisissant comme époux et épouse légitime. Dans les deux premiers codes, le missionnaire insistait beaucoup sur le fait que l'on prenait cette femme comme vraie femme et vice versa, ce qui laissait supposer que la monogamie était encore loin d'être la norme.

Dans le code de Huahine il était mentionné que le missionnaire procédait à l'enregistrement du mariage, et qu'il devait signer l'acte ainsi que les mariés et les témoins,

52 A la recherche de la Polynésie d'autrefois, (Paris, 1972) 586.

53 Ibid. 
ce qui n'était pas précisé auparavant. Ainsi, ces sociétés qui progressaient assez vite sur la voie de l'écrit, devaient désormais se familiariser avec la signature d'actes attestant de leur état.

La loi XIII «Concernant le refus de nourrir son épouse» était déjà présente dans les deux premiers codes, mais variait sensiblement quant au châtiment à infliger au coupable, puisque dans les deux codes antérieurs, il s'agissait de l'exil tandis que désormais seuls des travaux de construction de route ou de clôture sanctionnaient ce délit.

\section{Le concubinage $e^{54}$}

La loi XIX ne figurait pas dans les codes précédents et a été traduite par «De l'impudicité» d'après l'ouvrage de W Ellis, alors qu'il s'agissait «Du concubinage»:

Lorsqu'un homme vit en concubinage avec une femme, ceux-ci sont jugés et condamnés à des travaux: l'homme à la construction d'une route d'une longueur de cinquante brasses et d'une largeur de deux brasses; la femme à la confection de vêtements faits avec l'écorce de purau 55 dont deux seront destinés au roi et deux au chef de district, sinon à la confection de deux nattes, une pour le roi, et une pour le chef; sinon à la confection de grandes pièces d'étoffe en écorce battue, de quarante brasses de long pour le roi et 20 pour le chef.

Les missionnaires qui voulaient inculquer les valeurs de la morale chrétienne aux insulaires ne pouvaient tolérer le concubinage qui correspondait à une certaine marginalité par rapport à la norme qui était représentée par le mariage. De plus le concubinage était trop proche des unions du passé pour ne pas représenter un danger pour le modèle que les missionnaires souhaitaient édifier. Aussi était-il sévèrement sanctionné de travaux de construction pour les hommes et de tressage pour les femmes.

54 «L'Occident barbare connut des unions durables et acceptées par l'environnement social sans être régies par le droit, unions volontiers appelées, pour cette raison, «inférieures», à l'instar du concubinat de la Rome antique. Des documents de nature littéraire ou même canonique des siècles qui ont suivi les invasions y font allusion, sans d'abord les réprouver. Mais au cours du IX ème siècle, plusieurs conciles ont condamné le concubinat, sans bien sûr, le faire disparaître.» Bart J, Histoire du droit privé, supra n 49, 56.

55 Purau: arbre à fleurs jaunes poussant sur le littoral. 


\section{LOIS SUR LE SABBAT ET L'IMPÔT}

\section{A Sur le jour du Sabbat}

La loi VII «Concernant la non-observance de la journée du Sabbat» faisait déjà partie des deux premiers codes; mais dans celui de Huahine, elle est définie avec davantage de précision. $^{56}$

Le jour du sabbat devait être réservé au culte, et ceux qui ne respectaient pas ce commandement étaient sanctionnés. A ce sujet, W Ellis apporta quelques précisions: ${ }^{57}$

La loi qui interdisait de travailler le jour du sabbat est peut-être assortie d'une punition qui excède la faute commise; on doit toutefois remarquer que, en tant que nation, ils étaient habitués à strictement respecter un jour de repos pour des raisons religieuses avant même la publication d'une loi faite en vue d'éviter des désagréments à ceux qui désiraient consacrer un jour à des offices religieux. La route que les violateurs du sabbat avaient à construire n'était rien de plus qu'un petit sentier, avec une rigole creusée de chaque côté, sentier recouvert de sable ou de terre, pris sur les bords.

\section{B L'impôt}

La loi XXVI «Concernant les tributs à fournir au roi et aux chefs» apparaît pour la première fois dans le code E Ture No Huahine de 1822-1823.

Cette longue loi sur l'impôt obligatoire à verser au roi et aux chefs de district était justifiée par la parole divine. Quelques citations puisées dans la Bible permettaient d'en établir la nécessité et de l'envelopper du respect lié à celle-ci. Tout habitant de l'île devait

56 «C'est un grand crime devant Dieu pour un homme de travailler le jour du sabbat. Le travail qui ne peut être remis, tel que préparer de la nourriture quand un malade désire des aliments chauds et fraîchement préparés, est autorisé; mais il n'est pas permis d'accomplir des travaux tels que bâtir une maison, construire un bateau, cultiver la terre, pêcher et tout autre travail qui peut être remis au lendemain.

Personne ne devra voyager sur une longue distance le jour du sabbat. Pour ceux qui désirent assister au service, il faut qu'ils voyagent le jour de la cuisson des aliments (la veille).

S'il est impossible de voyager la veille, on peut le faire le jour même (pour assister au culte), mais il est interdit de marcher sur un long parcours (aller de village en village).

L'individu qui continuera à faire ainsi des choses interdites sera averti par les magistrats de ne pas le faire; mais s'il n'en tient pas compte, il sera obligé de travailler à la construction de la route $\mathrm{d}^{\prime}$ une longueur de cinquante toises et d'une largeur de deux toises. Et s'il continue à travailler un jour de sabbat, il fera un furlong $(201 \mathrm{~m})$ de plus.»

57 A la recherche de la Polynésie d'autrefois, (Paris, 1972) 588. 
participer à l'entretien du roi. L'impôt n'était pas requis pour la chose publique, mais pour l'intérêt du roi. ${ }^{58}$

La dernière loi du code «Concernant les nouvelles lois» édictait:

58 «Chaque terre qui a reçu la parole de Dieu et celle qui ne l'a pas reçue, dont les institutions sont valables, est d'accord pour dire qu'il est juste de fournir des provisions à son propre roi qui dirige le gouvernement et aux chefs de district.

Il y a également une chose fréquemment montrée dans la parole de Dieu et enseignée par Jésus, notre seigneur, quand il dit:

«Rendez à César ce qui appartient à César.»

Aussi, il est juste que nous fassions la même chose. Chaque individu devra participer au revenu du roi. L'homme qui possède plus doit fournir plus que l'homme qui possède moins. Les gouverneurs ou chefs de district devront donner deux cochons par an. S'ils n'ont pas de cochon, dix mesures d'arrow-root; sinon dix tonneaux de bambou remplis d'huile de noix de coco. Ces tonneaux doivent être de grande taille.

Les raatira, les fermiers et les petits propriétaires devront donner un cochon par an. S'ils n'ont pas de cochon, cinq mesures d'arrow-root; sinon cinq tonneaux d'huile.

Ceux qui ne possèdent pas de terre mais qui appartiennent à cette île ou appartiennent à une autre île, mais habitent sur celle-ci, devront fournir un cochon par an (plus petit que celui fourni par les fermiers), s'ils n'ont pas de cochon, trois mesures d'arrow-root ou trois tonneaux d'huile.

Les fermiers devront également préparer autre chose pour le roi qui dirige le gouvernement: chaque district devra préparer chaque année deux rouleaux de tissu, longs de dix toises et larges de deux; s'ils ne sont pas grands, une toise de fin tissu d'hibiscus, sinon trois toises de tissu indigène.

Telles sont les provisions pour les chefs que les fermiers doivent fournir pour une année: un cochon chacun, sinon, cinq mesures d'arrow-root ou cinq tonneaux d'huile. Et comme pour le roi, deux rouleaux de tissu devront être fournis par les habitants du district à leur propre gouverneur et qui devront être identiques en longueur et en largeur à ceux pour le roi. Si les rouleaux ne sont pas grands, un rouleau d'hibiscus ou trois toises de tissu indigène. Tel est le revenu que les districts devront fournir pour leur gouverneur chaque année, les habitants de chaque district pour leur propre gouverneur ou chef de district et telles sont les fournitures que les gouverneurs et la population devront donner au roi.

L'homme qui, à cause de maladie, ne peut fournir le revenu spécifié pour une année devra solliciter l'indulgence de la part du roi et des gouverneurs. Mais si c'est à cause de la paresse ou de toute autre chose qu'il ne le fait pas, il sera banni mais ne sera détenu par personne.

Il est juste que les fermiers agissent généreusement envers leur roi et leur gouverneur en donnant des provisions. Il faudra apporter au roi une partie des provisions telles que: fruits de l'arbre à pain, arum, bananes, ignames, et autre nourriture de ce genre. La nourriture doit être apportée crue. Le cochon pourra être remplacé par du poisson. Tout ceci devra bien se passer.» 
S'il survient un crime comparativement petit et qui n'est pas spécifié dans les lois, il est évident que ce code devra être modifié. Les lois devront être révisées ou corrigées annuellement.

A ce moment-là, devront être insérées les nouvelles lois concernant le crime qui aurait pu être omis avec la punition qui correspond pour que les coutumes de ce pays puissent être régulières et justes.

Les missionnaires, les chefs et la reine qui donna son accord mirent au point le troisième code de lois de la Polynésie, en tentant de remédier aux erreurs commises dans les deux premiers codes. Ces structures juridiques allaient servir de bases à tous les autres codes; avec pour objectif d'envisager le plus grand nombre possible de cas d'espèce, pour que la justice soit correctement administrée et que l'on s'éloigne des situations où régnaient l'arbitraire et le pouvoir discrétionnaire.

Il est intéressant de remarquer que dans notre Code civil, les juristes du début du XIX siècle ont d'abord privilégié les personnes dans le Livre premier, avant de s'intéresser aux biens et aux différentes modifications de la propriété dans le Livre deuxième, pour terminer dans le Livre troisième par les différentes manières dont on acquiert la propriété.

Les missionnaires protestants du début du XIXème siècle en Polynésie se sont d'abord préoccupés de l'interdit majeur et biblique que représente le meurtre dans la première loi. Puis le vol, les dégâts matériels causés par les cochons, la vente, les échanges, la journée du sabbat, la rébellion...ont fait l'objet de leur attention avant de s'intéresser aux personnes seulement à la loi IX avec la polygamie, puis le mariage.

Aucun titre ne fut consacré à l'état civil qui était pourtant une question complexe. Cela s'explique certainement par le fait que la vision de l'état civil des Polynésiens ${ }^{59}$ était peu choquante pour les Anglais ou au moins ne représentait pas un impératif en matière de législation.

\section{EN MATIÈRE DE PROCÉDURE}

Le code E Ture No Huahine était le plus complet en matière de procédure. Des lois pour les juges, le jury et les officiers de paix y étaient insérées après la trentième loi.

\section{A Lois pour les juges}

Elles se subdivisaient en deux parties, celles consacrées aux juges principaux et celles attribuées aux juges de second rang.

59 Les Polynésiens changeaient plusieurs fois de nom dans leur existence en fonction des grands événements de leur vie. 
(a) Concernant les juges principaux.

Ces lois étaient au nombre de quatre et avaient pour objectif d'établir des règles écrites quant à la nomination des magistrats, leurs obligations et leur salaire.

1- Le roi ou les gouverneurs suprêmes choisiront les juges principaux; et quand un juge meurt ou est destitué de ses fonctions, ou quand un juge part pour une autre île, le roi ou les chefs suprêmes doivent en nommer un autre pour qu'il puisse continuer la tâche entreprise.

2- Les devoirs des juges principaux (ou présidents). Tel est leur travail: quand un homme est jugé et que sa faute est parfaitement établie, le juge prononcera la sentence de son crime. La punition prévue par la loi qui correspond à ce crime sera prononcée et pas une autre.

3- Concernant la procédure: le juge écrira le nom du prisonnier, la faute qu'il a commise, le nom des personnes par lesquelles il est accusé et la punition qui lui a été infligée dans un livre qui pourra être consulté par le roi et la population.

4- Concernant le salaire: le salaire des juges présidents sera donné une fois par an par le roi. Toutes les confiscations appartiendront au roi sauf les cas spéciaux stipulés par les lois.

La rédaction de ces lois permettait une certaine transparence afin de prémunir les administrés contre l'arbitraire. L'élaboration d'un compte-rendu écrit qui pouvait être librement consulté par la population favorisait la publicité des débats.

(b) Concernant les juges de district.

Ces lois étaient au nombre de six et permettaient de structurer le champ d'application des droits et des devoirs de ces juges, largement sollicités.

1- Le roi ou les chefs suprêmes choisiront et nommeront les magistrats pour tous les districts.

2- Leurs devoirs: une personne accusée d'un crime quelconque, si le juge président n'est pas sur place, devra être amenée devant les magistrats du district qui jugeront ces personnes (dans leurs districts respectifs); ils devront également assister aux autres séances de jugements.

3- Quand un délit est commis, tel que le vol, ou offense similaire, la personne dont le bien a été volé, devra aller voir le magistrat et lui faire part de la chose. Le magistrat écrira les noms des accusés et de l'accusateur. Si la personne dont le bien a été volé ou qui a été attaquée désire que l'offenseur soit poursuivi, il sera jugé; sinon il ne devra pas être immédiatement jugé.

4- Les magistrats devront s'efforcer de calmer toutes les colères et les malentendus qui peuvent survenir, tels que les bagarres, les brouilles de famille, les disputes et les batailles pour que la paix soit maintenue. Il ne faut pas que les gens les traitent irrespectueusement.

5- Quand la sentence a été prononcée, le magistrat doit veiller à sa bonne exécution et diriger les messagers ou les officiers de paix. 
6- Il est du devoir du roi de fournir des rémunérations à tous les magistrats; ces rémunérations devront être faites annuellement pour la vigilance avec laquelle ils veillent sur 1'ordre public.

Ces magistrats de district semblaient être amenés à traiter des affaires courantes et à faire exécuter les peines avec l'aide des officiers de paix. Ils représentaient un premier degré de juridiction.

\section{B Le Jury}

Comme à Raiatea dans le code E Ture No Raiatea... de 1820, où cette institution fit son apparition, les lois concernant le jury étaient au nombre de quatre dans le code E Ture No Huahine, de 1822-1823.

1- Personne ne devra être jugé pour un grand crime ou délit sans jury. Il doit toujours y avoir un jury dans tous les jugements sauf ceux de moindre importance, telles que querelles et offenses.

2- Quand un homme est jugé pour un crime, le juge doit choisir six hommes pour former le jury; on devra choisir des hommes intègres; ils devront écouter très attentivement le dénouement ou les investigations du sujet. Quand l'examen des preuves est terminé, le jury se réunira en privé pour discuter de ce qu'ils ont entendu durant le procès, la défense de l'accusé et les accusations de l'accusateur avec les témoignages des témoins. S'ils pensent unanimement que la personne jugée est coupable, qu' elle a réellement commis le crime (il faut un témoignage identique de deux personnes), l'un d'entre eux s'adressera au juge en disant: «Cet homme est coupable.»

Alors le juge prononcera la sentence contre le criminel; on devra prononcer une sentence écrite dans la loi. Mais si le jury au complet pense que l'homme accusé n'est pas coupable, alors l'un d'entre eux dira: «Il n'est pas coupable.»

Si la personne jugée est un membre de la famille royale, alors le jury sera composé de membres de la famille royale (ou de personnes de rang égal); si c'est un propriétaire terrien ou un fermier qui est jugé, le jury sera composé de propriétaires terriens ou de fermiers.

3- Si durant le procès, un membre du jury veut poser une question au prisonnier ou à un témoin, il a le droit de le faire.

4- Si la personne accusée remarque parmi le jury une personne qu'il sait cruelle ou mal pensante ou un homme que son cœur n'approuve pas, il a le droit de dire au juge:

«Retirez cet homme et ne le laissez pas être un membre du jury.» Le juge devra alors chercher une autre personne pour la remplacer et continuera le procès de l'accusé. S'il y a deux ou trois personnes dans le jury que le prisonnier n'approuve pas, il faudra les changer; mais plus de quatre personnes ou la totalité ne peuvent être changées. Quand deux personnes sont renvoyées, les juges doivent en chercher deux autres. Ensuite il jugera les personnes accusées. 
L'utilisation d'un jury pour tout délit d'importance était une coutume juridique anglaise introduite aux Iles-sous-le-Vent par les missionnaires à Raiatea et maintenue à Huahine. Cette pratique judiciaire permettait d'élever un rempart contre l'arbitraire et le pouvoir discrétionnaire des juges et d'assurer à chaque prévenu un jugement équitable où les jurés appartenant à la même classe sociale que l'inculpé le déclaraient, après examen du dossier et récusations possibles, coupable ou non coupable.

\section{Les officiers de paix}

La procédure les concernant comprenait dans ce code deux lois; ils étaient aussi appelés les messagers des magistrats.

Concernant les messagers des magistrats.

1 - Leurs devoirs: tel est le devoir d'un messager ( ou officier de paix). Quand on accuse un homme et qu'il doit être conduit devant un magistrat, le magistrat doit envoyer un messager chercher l'accusé pour qu'il soit amené devant lui et gardé durant le procès. Quand le procès est terminé, le messager devra superviser l'exécution de la sentence prononcée par le juge ou le magistrat et gardera l'accusé d'une manière vigilante, jusqu'à ce que la sentence soit appliquée.

2 - Concernant la rémunération: le roi devra donner annuellement aux messagers les provisions dont ils pourraient avoir besoin en paiement du bon ordre qu'ils font régner sur l'île.

Ces messagers des magistrats semblaient détenir un pouvoir de police; ils étaient diligentés par les juges pour conduire un individu à comparaître devant eux, à le garder au cours du procès et à l'obliger à exécuter la sentence à l'issue du jugement.

Enfin, avant de s'achever, le code E Ture No Huahine, établissait une liste de huit lois intitulées «Directives pour les juges et le jury» L'intérêt de celles-ci se trouvait dans le fait qu'elle donnaient au code une volonté de rigueur, d'équité et de respect des libertés individuelles. En 1822-1823, l'île de Huahine fut dotée en matière juridique de la codification la plus avancée du Pacifique sud. ${ }^{60}$

60 «Directives pour les juges et le jury.

1 - Les juges et le jury ne devront pas faire attention aux conditions de l'homme. Si c'est un homme de grande influence, il faut le laisser être un homme de grande influence; si c'est un voisin, qu'il reste un voisin; si c'est un parent, qu'il reste un parent; si c'est un ami, qu'il reste un ami; tout ceci ne doit pas rentrer en cause. On doit considérer seulement ce qui est écrit dans les lois.

2 - Quand un délit est commis, tel que le vol ou l'adultère, si la victime désire que l'offenseur soit jugé, il devra aller lui-même voir le magistrat et présenter l'affaire devant lui. Le magistrat écrira 
Ces lois qui précisaient les règles de procédure à appliquer à chaque cas d'espèce laissaient apparaître un désir de rigueur et d'équité. Les traitements de faveur étaient proscrits pour les personnes influentes ou les proches; on demandait à faire vérifier les faits reprochés par un messager du magistrat, soit une enquête de police était organisée

son nom et le nom de la personne accusée pour que le procès soit légal. Mais si les offenses touchent toute l'île, tels que meurtre, rébellion, conspiration, non observance du sabbat, n'importe qu'elle personne sera compétente pour en informer le magistrat; celui-ci écrira son nom ainsi que le nom de l'accusé, il ne devra pas faire venir l'accusé d'après de vagues bruits. Il faut qu'un messager aille vérifier le fait sur place.

3 Quand une personne est amenée devant un tribunal et quand les magistrats sont rassemblés, l'accusé, l'accusateur et les témoins étant également présents, le magistrat ou le juge déclarera publiquement le crime dont l'offenseur est accusé et lui demandera alors si les accusations sont exactes. Si le prisonnier répond: «Oui, c'est exact», le juge prononcera la sentence. Si le prisonnier répond «Non, je n'ai pas commis ce crime», les juges demanderont alors à la personne sur les accusations de qui le prisonnier a été appréhendé de confirmer ses accusations. S'il y a deux témoins, on entendra les deux témoins; s'il y en a trois, on entendra les trois. Il faut que le témoignage soit clair et évident. Les juges demanderont alors au prisonnier s'il a quelque chose à dire. S'il y a une personne qui sait que le prisonnier est innocent, elle devra en témoigner; et s'il y en a deux, on entendra les deux; s'il y en a trois, on entendra les trois; ils doivent tous témoigner. Si la personne accusée désire poser des questions à l'accusateur, elle a le droit de le faire. Elle devra la poser au juge qui répétera la question à l'accusateur.

4 Nul homme ne peut être arrêté sans cause valable. Quand un cochon pénètre dans un jardin, le propriétaire du cochon ne doit pas être arrêté, mais on doit juste communiquer le fait au magistrat et il devra envoyer son messager chercher le propriétaire du cochon pour qu'il soit jugé en accord avec la loi III. Le même procédé devra être employé dans les offenses de moindre importance; mais pour le meurtre, le vol, la rébellion et tous les grands délits, on doit arrêter l'offenseur. L'emprisonnement ne doit pas être long avant la convocation du tribunal. Un, deux ou trois jours seront suffisants. Ne pas le faire plus longtemps.

5 Quand de petits délits sont commis, le magistrat du district pourra effectuer le jugement; pour des délits importants, les juges et le jury se rassembleront à un endroit pour le jugement.

6 Quand un homme est jugé par le magistrat du district et qu'il reçoit de lui la sentence, si la personne jugée considère que le juge a été injuste avec elle et qu'il a fait ainsi accroître la punition; s'il dit je veux aller devant le juge président et le jury pour être jugé, il a le droit de le faire. Il devra aller devant la cour suprême pour être jugé.

7 Quand un homme est jugé, accusé et condamné par le juge et le jury, il ne doit pas être battu avec un bâton, transpercé par une lance ou subir tout autre outrage. La punition appropriée devra être appliquée.

8 Quand un homme est accusé d'un délit grave par le juge et le jury et qu'ils pensent unanimement qu'il mérite une punition, le juge devra alors écrire son délit sur un papier, sa propre décision et la décision du jury en ce qui concerne la sentence. Ceci doit être apporté au roi et si le roi approuve leur décision, il devra écrire sur le papier apporté par le juge: «Approuvé pleinement» et écrire son propre nom en dessous et alors seulement la punition pourra être infligée à l'offenseur. Si le roi désire alléger la sentence, il peut le faire mais il ne peut l'augmenter. 
afin d'étayer les allégations des accusateurs, et l'on accordait de l'importance aux témoins, que l'on tenait à entendre.

Les petits délits étaient jugés comme à Raiatea par une sorte de tribunal de police du district. L'on avait le droit de faire appel d'un jugement auprès du juge président et du jury. Une juridiction de second degré existait pour favoriser le droit de recours des plaignants et leur garantir une plus grande justice.

Comme dans le code E Ture No Raiatea...de 1820, le respect des libertés individuelles était assuré par le rejet des arrestations arbitraires et l'incarcération devait être de courte durée avant le jugement. L'esprit de l'habeas corpus flottait aussi sur le code E Ture No Huahine, avec interdiction de maltraiter le coupable.

Enfin, comme à Raiatea, le roi ou la reine détenait un droit de grâce, et devait dans les cas graves se prononcer sur la sentence qui était infligée par les juges. Il pouvait l'alléger et non l'aggraver, ce qui montrait un respect envers les magistrats et la justice qui ne pouvait être détournée par la volonté royale.

Le missionnaire William Ellis cita dans son ouvrage le cas d'une affaire qui se déroula en 1822 et qui illustre toutefois les difficultés que les personnages influents pouvaient avoir à se soumettre aux nouvelles lois.

Il écrivit qu'à l'automne 1822 la reine de Tahiti, la veuve de Pomare, visita Huahine. ${ }^{61}$ La suite qui l'accompagnait depuis Tahiti, ayant besoin d'une pièce de bois, la reine leur dit de couper un arbre à pain poussant dans le jardin d'un pauvre homme, sur le côté opposé à la baie, près de laquelle était située sa résidence.

Ses ordres furent exécutés et l'arbre fut emporté. Teuhe, le propriétaire de la terre sur laquelle se trouvait l'arbre, revenant le soir dans sa maison, vit les traces du passage des ravisseurs: la souche saignait encore, les branchages étaient éparpillés sur le sol et le tronc majestueux avait disparu. Mis au courant par ses voisins que c'étaient les gens de la Reine qui l'avaient coupé, il partit voir le magistrat du district et déposa une plainte contre Sa Majesté la reine. Le magistrat lui dit de revenir au tribunal public le lendemain matin au lever du soleil et de justifier sa plainte. Il envoya ensuite son serviteur vers la reine et demanda sa présence à la même heure.

Le lendemain matin, comme le soleil se levait au-dessus de l'horizon, on vit Ori le magistrat assis en plein air sous les branches d'un arbre vénérable. Devant lui sur une natte finement tressée était assise la reine entourée de sa suite; à côté d'elle se tenait debout le paysan indigène et tout autour ce que l'on pourrait appeler les officiers de police.

61 Il devait vouloir parler de la reine de Huahine, Teri'itaria. 
Se tournant vers Teuhe, le magistrat lui demanda pour quelle raison il était venu. Le pauvre homme dit que dans son jardin poussait un arbre à pain dont l'ombre était bénéfique pour les habitants de sa maison et dont les fruits nourrissaient sa famille cinq à sept mois de l'année. Hier, quelqu'un avait coupé l'arbre. On lui avait dit que c'était sur l'ordre de la reine. Il savait qu'il existait des lois et il pensait que ces lois protégeaient la propriété des pauvres gens aussi bien que celle des chefs et des rois; il désirait savoir s'il était juste, qu'à son insu et sans son consentement, l'arbre ait été coupé.

Le magistrat se tournant vers la reine lui demanda si elle avait ordonné de couper cet arbre. Elle répondit: «Oui». Il lui demanda alors si elle ne savait qu'ils avaient des lois. Elle répondit: «Oui» encore, mais qu'elle ignorait que les lois s'appliquaient à elle. Tenant en ses mains un exemplaire des lois, le magistrat demanda:

«Est-ce que dans ces lois-là il y a des exceptions concernant les chefs, les rois ou les reines ?» Elle répondit «Non» et dépêcha un serviteur dans sa maison pour qu'il lui ramène une bourse remplie de dollars qu'elle jeta devant le pauvre homme en compensation de sa perte. «Arrêtez, dit le juge, ce n'est pas encore terminé.» La reine se mit à pleurer.

«Croyez-vous qu'il ait été juste que vous coupiez cet arbre sans demander la permission au propriétaire ?» continua le magistrat.

«Ce n'était pas juste» dit la reine. Puis se tournant vers le pauvre homme, il demanda:

«Quel dédommagement demandes-tu ?»

Teuhe répondit: «Si la reine se rend compte qu'il n'était pas juste de prendre un arbre à un pauvre homme sans sa permission, je suis sûr qu'elle ne recommencera pas. Je suis satisfait»

Il ne demanda nulle autre récompense. Son désintéressement fut applaudi et l'assemblée se dispersa. Le missionnaire ajouta que la reine lui fit envoyer à titre privé un cadeau égal à la valeur de l'arbre. ${ }^{62}$

Cet exemple illustre bien le fait que cet effort de codification gardait un caractère abstrait, et que si l'homme du peuple y trouvait le moyen de faire valoir ses droits, la reine en revanche bien qu'ayant approuvé le code, ne se sentait pas concernée par celui-ci.

Le premier code de lois E Ture No Huahine de $1822-1823^{63}$ représentait le recueil juridique le plus accompli du Pacifique sud. Il fut ensuite perfectionné dans les décennies qui suivirent, par les codes de 1853 et 1886.

62 Ellis W, A la recherche de la Polynésie d'autrefois (Paris, 1972) 593-594. 
On March 5, 1797, the first missionaries of London Missionary Society arrived in Tahiti, by the ship the "Duff". At the beginning of 19th century, starting in Tahiti and Moorea, they began the evangelisation of the population, moving then to the Leeward Islands.

With the progression of Christianity, the old religion gradually disappeared changing the society's references. King Pomare II knew how to benefit from the missionaries' influence in order to increase his authority on the local populations formerly subjected to many scattered authorities. In return the missionaries, using Pomare's powers, were able to carry out the evangelisation. Quickly, appeared the need to write codes of laws, based on good conduct principles and inspired by the wording of the Bible that the missionaries translated into the vernacular language and made available for the local populations. These codes provide the last indication of what were the customary laws, in a society of oral tradition in full upheaval. In 1819 King Pomare II promulgated the 19 articles of the first code of laws in Polynesia: the code E Ture No Tahiti. In 1820, King Tamatoa in the Leeward Islands enacted the 25 articles of the code E Ture No Raiatea E no Tahaa E No Porapora E No Maupiti. In 1823, Teri'itaria, Queen of Huahine, daughter of Tamatoa King of the Leeward Islands and sister-in law and concubine of Pomare II promulgated the first code of laws of the island of Huahine drafted by the missionaries with the help of some customary local chiefs. It comprised 30 articles and represented in Polynesia, the most elaborate piece of legislation available at the beginning of the 19th century.

63 Les premiers codes de lois promulgués aux îles du vent et sous-le-vent entre 1819 et 1822-23, servirent de modèle à tous les autres codes réalisés ultérieurement, en Polynésie. Ils comportaient certains fondements des futures juridictions tahitiennes. Sage Y-L, (1997) 3 RJP. 